How should donors give foreign aid?

Project aid versus budget support 


\title{
How should donors give foreign aid? Project aid versus budget support*
}

\author{
Izabela Jelovac ${ }^{\dagger}$ \\ and Frieda Vandeninden
}

April 2008

\begin{abstract}
We develop a theoretical model to compare the two major foreign aid modalities: project aid and budget support. These two modalities have a different impact on the production of 'developmental goods'. Firstly, conditionality can be associated with budget support, but only a subset of the developmental expenses - the observable ones - can be subject to conditionality. Secondly, when using project aid, the donors control the overall allocation of the aid resources. However, we consider that, because of limited harmonisation and coordination, project aid can be associated with a cost of imperfect fit. We develop a unified framework to compare these two modalities where we allow the simultaneous utilisation of both instruments. We show that all the aid should be given via budget support, no matter whether conditionality is used or not. Furthermore, we show that the optimal use of conditionality depends on the recipient's developmental preferences, the productivity of the inputs and the level of aid compared to the recipient's budget: when these parameters are relatively high, conditionality should be enforced. Otherwise, the optimal aid allocation is such that all the aid is given through unconditional budget support. We conclude that conditionality does not always improve the aid effectiveness.
\end{abstract}

JEL Classification: F35, O19, D82

Keywords: Foreign Aid, Optimal Contract, Conditionality

\footnotetext{
* The authors gratefully acknowledge the comments of Axel Gauthier, Mathieu Lefèbvre, Pepita Miquel-Florensa, seminar participants at the MGSoG, participants to the II Guanajuato Workshop for Young Economists (Guanajuato, 2007) and to the annual meeting of Association of the Southern European Economic Theorists (Padova, 2007).

${ }^{\dagger}$ University of Lyon, Lyon, F-69003, France; CNRS, UMR 5824, GATE, Ecully, F-69130, France; ENS LSH, Lyon, F-69007, France ; Centre Leon Berard, Lyon, F-69003, France.

* MGSoG, Universiteit Maastricht. Frieda.Vandeninden@governance.unimaas.nl.
} 


\section{Introduction}

The effectiveness of foreign aid has been a major concern for the past decade. Several empirical studies have shown the weak impact of aid on poverty reduction and growth (Boone, 1996; Easterly, 2003, 2006; Hansen and Tarp, 2001, Burnside and Dollar, 2000; Collier and Dollar, 2002). ${ }^{3}$

The causes often pointed out are weak institutional capacities, corruption and the fungibility problem: all of these problems are present in many developing countries. Because of this observation - that aid is not efficient - aid modalities have also been criticised. In fact, apart from the inherent deficiencies of developing countries, the inefficiency could also come from the instruments chosen to deliver the foreign aid. The two main instruments (or modalities) are project aid (PA) and budget support (BS). Project aid involves the direct participation of the donors in the design and the implementation of a developmental project. With budget support, the donors provide support through the recipient government budget. ${ }^{4}$ Donors, in the case of budget support, can also impose conditionality on how to allocate the available resources.

These two instruments have a very different impact on development, their respective pros and cons have been largely analysed in the literature. First, we should note that the project aid has been the major aid modality since the 1950's. This trend can be explained by the belief that the main constraint to development was a lack of investment, a problem which could be overcome by channelling capital investment to developing countries. However, these off-budget funds have been more and more criticised over time, with regards to their poor impact on development: the main issues are a lack of coherence (between the individual projects of each donors and also with the national policies of the recipient country), no building of institutional capacities (because the donors do not use the national procedures), lack of transparency, risk of double use of resources (e.g. two schools are built in the same village) and high transaction costs (World Bank, 1998; Lavergne, 2003, Tarp, 2002). In the late 1990's, because of the weaknesses of the project aid, the donors' community started to consider the need for changes in the aid delivery system. There are

\footnotetext{
${ }^{3}$ Yet the literature on the impact of aid on growth and poverty reduction does not reach an agreement. See Rajan and Subramanian (2005) for a survey.

4 'The general characteristics of budget support are that it is channelled directly to partner governments using their own allocation, procurement and accounting systems, and that it is not linked to specific project activities' (OECD, 2007).
} 
five principles underlying the 'new aid system delivery', also called programme-based approach: 'ownership, alignment, harmonisation, managing for results, and mutual accountability.' (OECD, 2005). ${ }^{5}$ One of the aid modalities that best reflects these new principles is budget support. Budget support can take different forms: general budget support (funds mixed with recipient's general budget), sector budget support (e.g. funds transferred to health ministry) and conditional budget support (the aid is still injected in the recipient budget but the donors specify how the funds should be allocated). Even if the part of the budget support in the total worldwide aid allocation is not yet significant, the international community considers budget support as a promising aid modality: 'budget support avoids many of the problems that accompany other forms of aid (e.g. uncoordinated projects that undermine government systems, impose high transaction costs and lack sustainability). It tends to enhance country quality level of aid as a whole' (OECD,2007). ${ }^{6}$ Next, according to the OECD (2007), budget support should preferably be introduced in highly aid-dependant countries, when the recipient is a credible partner (e.g. in term of its commitment to a poverty reduction strategy) and when there is a certain level of governance and macroeconomics management. Consequently, more and more donors are emphasizing budget support as a way of financing development to complement or substitute for project aid (World Bank, 2005).

When comparing aid modalities, one should be aware of the different dimensions of the aid instruments. We can distinguish four dimensions: the type of finance (grants or loans), the procurement conditions (if aid takes the forms of materials, skills or money, and if it is tied on particular source on supply), the targeting of the resources (if the aid is ex-ante assigned to a particular use) and the disbursement channel (through or outside government budgeting). When comparing budget support and project aid, it is clear that we focus on the disbursement channels and, to a certain extend, on the targeting of resources (when considering conditional budget support).

However, while the (small) theoretical literature on aid allocation has recently grown, there are, to our knowledge, few attempts to compare these two instruments in a single formal model.

\footnotetext{
5 'Programme-based approach share the following features: leadership by the host country or organisation; a single comprehensive programme and budget framework; a formalised process for donor co-ordination and harmonisation of donor procedures for reporting, budgeting, financial management and procurement; Efforts to increase the use of local systems for programme design and implementation, financial management, monitoring and evaluation' in Harmonising Donor Practices for Effective Aid Delivery' (OECD, 2005).

${ }^{6}$ General budget support represented 5\% of the total aid or some $\$ 5 \mathrm{bn}$ in 2004, but it goes up to $45 \%$ of total aid in Uganda and reach some similar proportion in other sub-Saharan countries (OECD, 2007).
} 
Svensson (2000 and 2003) analyses the rent-seeking problem when there are competing social groups in a developing country and the time inconsistency problem when aid is conditional to policy reforms. Azam and Laffont (2003), focusing on ex-ante optimal contracts to avoid free riding, show how aid contacts can induce the recipient to reveal his preferences towards development. The paper most closely related to our model is Cordella and Dell'Ariccia (2007). It compares the respective advantages of budget support and project aid. They find that conditional budget support is always preferable to unconditional budget support. Moreover, project aid is more efficient than budget support when the developmental preferences of the recipient are high and when the total aid available is small relative to the recipient's budget.

We develop a theoretical model that compares the two modalities, according to the countries specificities: the recipients are heterogeneous while the donors are considered as an unified entity. We want to determine the optimal aid allocation contract. Such a model has already been developed by Cordella and Dell'Ariccia (2007). As in their paper, we consider that donors want to maximise the production of a developmental good, whereas the recipient (the government of a developing country) also cares about a non-developmental good and that only a subset of the developmental expenses can be subject to conditionality. In fact, we consider that not all government activities are monitorable. While they compare the two modalities in two separate models, we consider a unified framework in which the utilisation of both instruments is allowed. In reality, the recipient countries receive aid both via BS and PA (there is no case in which only one modality is adopted).

We proceed in two steps: in the first we do not allow conditionality and in the second we do. ${ }^{7}$ In the first case, we show that the unconditional budget weakly dominates project aid: if we consider that project aid funds are perfect substitutes for recipient's own funds (that is no loss of efficiency is associated with project aid), then the donors are indifferent between PA and unconditional BS (we can have a mixed contract). But if donors funds are not perfect substitutes for recipient's ones (e.g. because of imperfect fit of the project, lack of coordination and double use of resources), budget support always yields a higher level of production of developmental good, so that the optimal contract is to give aid only though BS. In both cases, the optimal aid contract leads to a distortion in the resources allocation because only a subset of the foreign aid

\footnotetext{
${ }^{7}$ We consider that conditionality can be only associated with budget support and it consists in setting a minimum level on some recipient's budget component (as in Cordella and Dell'Ariccia, 2007).
} 
reaches the developmental sector. Two major problems underlie this inefficiency: the crowding out effect of PA (also called the fungibility problem) and conflict of interest between donors and recipients (e.g. different developmental priorities). We then look at whether conditionality can reduce the inefficiency. If we allow conditionality with BS, the optimal aid allocation depends on the recipient's preferences. More specifically, we show that the conditionality does not always increase the production of the developmental good. It only does so when the donors and recipient's preferences are relatively close. In that case, conditional budget support is the optimal foreign aid allocation. When the preferences are relatively far apart, conditionality is not optimal. The optimal contract in that case is the same as the one in the non-conditional case. Then, the inefficiency in resources allocation remains the same. These findings are in accordance with a quote of the former chief economist of the World Bank François Bourguignon in Le Monde (16/02/2008): 'the countries where aid is the less efficient because their difficulties to use it are the most in need. Imposing them severe conditions to obtain the aid would be equivalent to not helping them. And this is worst than all'.

There are two major differences with the findings of Cordella and Dell'Arriccia (2007). First, the optimality of the conditionality depends on the productivity of the production factors, the developmental preferences and the ratio between aid and recipient's revenue while in Cordella and Dell'Arriccia, the conditionality is always preferred. This difference stems from the assumptions on the production function. We allow for variable productivity of the inputs of the developmental good while Cordella and Dell'Arriccia consider a symmetric production function. We add another dimension to the analysis because the productivity of the inputs can be interpreted as the degree of transparency: when there is a high degree of transparency, more inputs are observable, which increases the productivity of the observable ones. We find that more recipients accept the conditionality when the transparency is low. Additionally, the optimality depends on the aid dependency ratio: when the recipient becomes more aid dependent, the need for conditionality increases. The second difference concerns the optimality of project aid. In our framework, project aid can be the optimal contract only in the specific case of low developmental preferences coupled with perfect substitutability between donors and recipient's resources. In this particular case, the donors are indifferent between giving aid through unconditional budget support or project aid. 
The paper is structured as follows. The model is described in Section 2. Section 3 presents two extreme benchmark cases: One in which no aid is given and another in which the donor and the recipient share the same objective. In Section 4, we derive and discuss the optimal aid modalities assuming that the recipient and the donor do not need to share the same developmental objectives. Section 5 concludes. Proofs are in the Appendix.

\section{The model}

In our stylized framework, a developing country cares about the production of a developmental good, $s(k, e)$, that depends on an observable input, $k$ (capital goods), and a nonobservable input, $e$ (effort, administrative and managerial outlays, anything that is not observable to a donor). We assume a Cobb-Douglas production function for this developmental good: $s(k, e)=k^{1-a} e^{a}$ where $a(0 \leq a \leq 1)$ represents the productivity of the non-observable component of the development good production function. To a certain extent, the parameter $a$ can be interpreted as reflecting the level of transparency in the developing country: when the transparency is low, more inputs are therefore non-observable, which decreases the productivity of the observable ones. The developing country also cares about a non-developmental good, $m$. The preferences of the developing country for the developmental good and the nondevelopmental one are represented by a Cobb-Douglas utility: $V(m, s)=m^{1-\alpha} s^{\alpha}$, where $\alpha$ $(0 \leq \alpha \leq 1)$ represents the recipient's developmental preferences (if $\alpha=1$ he only cares about the developmental good).

To produce goods $m$ and $s$, the government of the developing country has at its disposal a tax revenue $G$ (exogenous and observable to the donor) and a transfer (aid) given by the donor.

The total aid available is denoted $T$ (exogenous). The aid can be given through two modalities: project aid and budget support. The donors have to decide how to spread out the total aid $T$ such that the level of developmental good is maximised (we consider that they only care about the developmental sector). The share of aid allocated via PA and BS is denoted as $A$ and $B$, respectively. The resources given via BS are simply added to the recipient's budget $G$. Moreover, still in the case of BS, the donors can also decide to impose the capital level $(k)$ that the recipient has to carry out in order to receive the BS. This reflects the conditionality. 
When PA is used, the resources $A$ do not go to the recipient budget, but are fully controlled by the donors. This allows us to consider the resources given through project aid (A) as an observable variable and then to add the resources $A$ to the capital level $k$ in the developmental goods production function. However, when eluding the national procedures, the project could not perfectly fit with the recipient poverty reduction strategy. So, the project could have a lower impact than if it were undertaken by the recipient. In the model, there is thus an efficiency loss due to the imperfect fit of the project: only a share $\gamma$ of the resources given via PA has an effective impact in the production of the developmental good, with $0 \leq \gamma \leq 1$ (if $\gamma=1$, there is no efficiency loss). The amount of resources $(1-\gamma) A$ represents the cost of imperfect fit.

We assume that the donors only care about the developmental good level of the recipient. This assumption could be interpreted as if we assumed paternalistic behaviour from the donors. However, we do not want to enter into this debate, and this hypothesis only means that the donors have their own developmental preferences. The government may have a different view about the priorities, this does not necessarily mean that their preferences are better or worse than the donor's ones. An expense could be crucial for the government (to improve civil servants' working conditions) without having a direct impact on poverty reduction for instance. The utility function of the donors consequently takes into account only the developmental good production function $s(k, e)$. Finally, we consider that the donors allocate the whole resources available $T$.

To sum up, the timing of the model is the following:

- The donor observes the preferences and budget of the government and then decides how to allocate the total aid available, $T$, between $A$ and $B$. If we allow for conditionality, the donor decides whether to use it and the level of resources that the government has to dedicate to the observable input $k$.

- The government observes the aid allocation and then decides how to spread his total available resources between the two inputs needed to produce the developmental good and the non-developmental good. If the budget support is conditional, then the government decides on the level of the non-observable input and the level of the nondevelopmental good, $m$. In addition, he chooses whether or not he respects the conditionality. 
In what follows, we provide a benchmark that expresses the optimal allocation when no aid is given. We further determine the first best (when the preferences are perfectly aligned) and the second best aid contract when:

(i) Conditionality is not possible;

(ii) Conditionality is allowed.

\section{The benchmark}

In the absence of aid, the government allocates his budget $G$ between $k, e$ (the two production factors for the development good) and $m$ (the composite good representing the nondevelopmental goods) so as to maximise his objective function (1) subject to his budget constraint (2):

$$
\begin{array}{ll}
\underset{k, e, m}{\operatorname{Max}} & V(m, s(k, e))=m^{(1-\alpha)} e^{a \alpha} k^{\alpha(1-a)} \\
\text { s.t. } & k+e+m \leq G
\end{array}
$$

Our problem is concave. Therefore, the first order conditions (F.O.C.) give us the optimal level of resources dedicated to the capital component, the non-observable component and to the sectors other than development:

\section{Result 1:}

$$
\begin{aligned}
& k_{N A}^{*}=\alpha(1-a) G ; \\
& e_{N A}^{*}=\alpha a G ; \\
& m_{N A}^{*}=(1-\alpha) G ; \\
& s_{N A}^{*}=\alpha a^{a}(1-a)^{1-a} G ; \\
& V_{N A}^{*}=\alpha^{\alpha}(1-a)^{\alpha(1-a)} a^{\alpha a} G .
\end{aligned}
$$

The results are intuitive: the more the recipient cares about development, the more resources are dedicated to developmental production factors and the less to the composite good. In other words, if the developmental preferences are high, few resources will be allocated to the non- 
developmental sector. The repartition between $k$ and $e$ also depends on their respective productivity. More resources will be allocated to the more productive factor.

We now consider that the donor gives the aid and we determine the first best allocation. We consider a hypothetical first best, where the production of the developmental good is maximised. Therefore, in our first best, the preferences of the recipient are perfectly aligned with those of the donors:

$\underset{A, B}{\operatorname{Max}} s(k+\gamma A, e)$ s.t. $k+e \leq G+B$ and $A+B=T$.

The effectiveness is therefore represented by a situation where no resources from the aid budget are diverted to the production of a non-developmental good. This is achievable only when the preferences are perfectly aligned. In that case, the optimal contract is such that all the aid is given indifferently through budget support and project aid (if there is no loss of efficiency associated with PA). But for every $\gamma<1$, PA leads to a lower level of developmental good than BS. Then the optimal contract is such that all the aid is given by BS.

The solution is therefore:

\section{Result 2:}

$$
\begin{aligned}
& k_{F B}^{*}=(1-a)(G+T) ; \\
& e_{F B}^{*}=a(G+T) ; \\
& m_{F B}^{*}=0 ; \\
& s_{F B}^{*}=(1-a)^{(1-a)} a^{a}(G+T) ; \\
& V_{F B}^{*}=s_{F B}^{*} ; \\
& \text { with } B^{*}=T \text { and } A^{*}=0(\text { for } \gamma<1) .
\end{aligned}
$$

In the first best case (when $\alpha=1$ ), the aid is perfectly effective: no aid is diverted to the nondevelopmental sector and the production of developmental good is maximized.

\section{The second best analysis}


The First Best cannot be achieved for every $0 \leq \alpha<1$, that is, if the recipient's preferences are not perfectly aligned with those of the donors. In that case, there is a conflict of interest between the donors and the recipient.

\subsection{Conditional budget support is not available}

The donor chooses how to allocate the aid (PA or BS) in order to maximize the level of developmental good whereas the recipient fixes the level of $k, e$ and $m$, once the aid allocation is observed.

The problem has the following form:

$$
\begin{aligned}
& \text { Donors: } \quad \operatorname{Max}_{A, B} \quad s(e, k+\gamma A)=e^{a}(k+\gamma A)^{1-a} \quad \text { s.t. } \quad A+B=T \\
& \text { Recipient: } \quad \operatorname{Max}_{k, e, m} V(m, s(e, k+\gamma A))=m^{(1-\alpha)} e^{a \alpha}(k+\gamma A)^{(1-a) \alpha} \quad \text { s.t. } k+e+m \leq G+B
\end{aligned}
$$

And such that every variable is positive (these non-negativity constraints are implicit in the remaining of the paper). We solve the problem by backward induction. We thus consider the maximisation problem of the recipient first.

\section{The recipient's maximisation}

From the F.O.C., we obtain the optimal level of $k_{N C}^{*}, e_{N C}^{*}$ and $m_{N C}^{*}$ (the subscript $N C$ holds for No Conditionality):

$$
\begin{aligned}
& k_{N C}^{*}=\alpha(1-a)(G+B)-(1-\alpha(1-a)) \gamma A \\
& e_{N C}^{*}=\alpha a(G+B+\gamma A) \\
& m_{N C}^{*}=G+B-e_{N C}^{*}-k_{N C}^{*}=(1-\alpha)(G+B+\gamma A)
\end{aligned}
$$

The level of the capital good $k_{N C}^{*}$ is decreasing in $A$ while it is increasing in $B$. When project aid increases, the optimal capital level decreases because the recipient, for every $0<\alpha<1$, reallocates some funds from the budget $G$ to the non-developmental sector. The PA has a negative incentive effect on the implication of the government in the developmental sector 
financing. In fact, having observed that the donors finance themselves the developmental sector, the recipient can re-assign some funds that would have been allocated to $k$ if no aid were given. There is thus a crowding out of the aid, which reflects the fungibility problem.

However, when we examine the level of $m$, we see that it is increasing in both $A$ and $B$ but the PA leads to a weaker allocation of resources to the non-developmental sectors than the BS:

$$
\frac{\partial m_{N C}^{*}}{\partial A}=\gamma(1-\alpha)<\frac{\partial m_{N C}^{*}}{\partial B}=1-\alpha, \text { for every } 0<\gamma<1 .
$$

In fact, since the project aid funds $A$ are not transferred to the budget $G$, the recipient cannot absorb all the resources $A$. In that respect, we can say that the BS leads to a worse distortion in resources allocation. Nevertheless, $s_{N C}^{*}=\left(k_{N C}^{*}\right)^{1-a} \cdot\left(e_{N C}^{*}\right)^{a}$ is increasing in both $A$ and $B$ (and more in $B$ than in $A$ if $\gamma<1)$.

\section{* Donors' maximization}

We assume that the donors care only about the level of development good. The total budget available is exogenously given and allocated entirely (so that the budget constraint is binding). They consequently maximise the level of development good:

$$
\underset{A, B}{\operatorname{Max}} \quad s(e, k+\gamma A)=e_{N C}^{* a}\left(k_{N C}^{*}+\gamma A\right)^{1-a} \text { s.t. } \quad A+B=T
$$

There is no interior solution for the maximisation problem. We have, for every $\gamma<1$, a corner solution: $A_{N C}^{*}=0$ and $B_{N C}^{*}=T$. That is, the donors give all the aid through budget support and do not use project aid. When $\gamma=1$, then PA has exactly the same impact as BS on the level of developmental good. In that case, the donors are indifferent between PA and BS. ${ }^{8}$ The optimal levels of resources allocation when there is no conditionality are thus:

\section{Result 3:}

$$
\begin{aligned}
& k_{N C}^{*}=\alpha(1-a)(G+T) ; \\
& e_{N C}^{*}=\alpha a(G+T) ;
\end{aligned}
$$

\footnotetext{
${ }^{8}$ Moreover, in order to respect to non-negativity constraint, the recipient is indifferent between PA and BS only if $A^{*} \leq \frac{\alpha(1-a)}{1-\alpha(1-a)}\left(G+B^{*}\right)$. If not, BS dominates PA even if $\gamma=1$.
} 


$$
\begin{aligned}
& m_{N C}^{*}=(1-\alpha)(G+T) ; \\
& s_{N C}^{*}=\alpha a^{a}(1-a)^{1-a}(G+T) ; \\
& V_{N C}^{*}=a^{\alpha a}(1-a)^{\alpha(1-a)}(1-\alpha)^{\alpha(1-\alpha)}(G+T) ; \\
& A_{N C}^{*}=0 \text { and } B_{N C}^{*}=T .
\end{aligned}
$$

Proposition 1: Budget support weakly dominates project aid, when conditionality is ruled out.

When it is not possible to condition the level of observable input to the disbursement of BS, the optimal aid contract is such that all the aid is given via BS. This is the consequence of the crowding out effect of the project aid: the recipient observes that the donors finance the developmental good and therefore decreases the resources he would have allocated without any aid. But if the project aid funds are perfect substitutes for the recipient's ones, the donors are indifferent between PA and BS (however, the proportion of PA has to be restricted in order to satisfy the non-negativity constraint). In both cases, the optimal aid contract leads to a distortion in resources allocation: a part of the aid, $(1-\alpha) T$, is allocated to the non-developmental sector. When considering the realistic hypothesis of imperfect fit of PA, we have seen that the impact of the fungibility coupled with the misalignment of the preferences leads to a poor improvement of the development. Even if more aid funds are allocated to the non-developmental good with BS, the donors, while maximising the production of the developmental good, proposes a contract with only budget support.

To reduce the inefficiency due to the high resources diversion, the donors could introduce conditionality over the observable component. We now turn to the case of PA and BS associated with conditionality.

\section{2. $\quad$ Aid with conditionality}

As previously stated, the production of a development good depends on an observable component (the capital level $k$ ) and an unobservable one $(e)$. Therefore, the donors can impose the level of capital when giving the aid through BS. The conditionality is thus only associated with BS. If the aid is given only through PA, the level of capital is decided by the recipient as above. It could 
seem simple to define conditionality as a minimum level of expenses, but in the framework developed here, it does reflect the limited capacities of the donors to monitor all recipient's expenses. This is evidently a major shortcoming of the Budget Support.

We proceed as before to determine the optimal allocation of aid.

\section{* The recipient maximisation problem}

The aid given via BS is transferred to the recipient only if the latter respects the conditionality, that is, if the resources allocated to the observable input are at least as high as the level decided by the donors: $k \geq \tilde{k}$. Therefore, the recipient chooses the level of resources allocated to each input knowing that if the level of resource allocated to the observable component is inferior to the conditional level, he does not receive any budget support. The maximisation problem has thus the following form:

$$
\begin{aligned}
& \underset{k, e, m}{\operatorname{Max}} V(m, s)=m^{(1-\alpha)} e^{a \alpha}(k+\gamma A)^{(1-a) \alpha} \quad \text { s.t. } k+e+m \leq G+B, \quad \text { with } \\
& \left\{\begin{array}{l}
B>0 \text { if } k \geq \tilde{k} \\
B=0 \text { if } k<\tilde{k}
\end{array}\right.
\end{aligned}
$$

To understand the influence of the conditionality $\tilde{k}$ on the recipient's behaviour, we have to take into account the individual rationality constraint (IR) of the recipient: the latter accepts the conditionality if and only if his utility level when no BS is given $\left(V_{0}\right)$ is smaller than the utility level when BS is granted $\left(V_{C}\right): V_{C} \geq V_{0}$. We can determine the critical level of capital, denoted $k_{M}$, that satisfies the equality of the two utility levels: $V_{C}\left(k_{M}\right)=V_{0}\left(k_{0}^{*}\right)$, with $k_{0}^{*}$ being the best respond level of observable input when no budget support is granted. More precisely, $k_{M}(a, \alpha, \gamma, A, B, G)$ is such that:

$\alpha(1-a) \ln \left(k_{M}+\gamma A\right)+(1-\alpha+\alpha a) \ln \left(B+G-k_{M}\right)=\alpha(1-a) \ln \left(k_{0}^{*}+\gamma A\right)+(1-\alpha+\alpha a) \ln \left(G-k_{0}^{*}\right)$.

Consequently, we can distinguish three cases (Figure 1 hereunder helps identifying those three cases). 


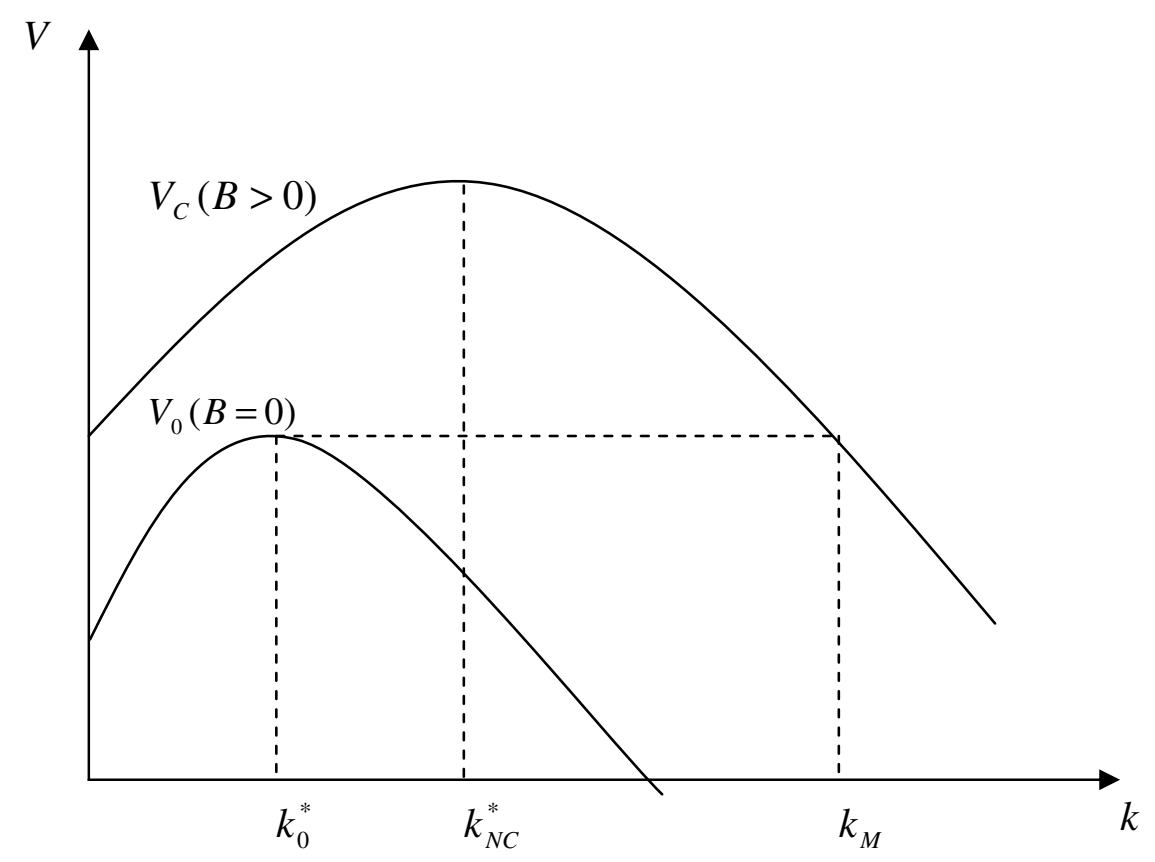

FIGURE 1

a. when $\tilde{k} \geq k_{M}$ : the conditional level does not satisfy the IR. The recipient is therefore better off allocating the amount of capital $k_{0}^{*}$, which maximises his utility when no BS is granted: $k_{0}^{*}=\alpha(1-a) G-(1-\alpha+\alpha a) \gamma A$.

b. when $\tilde{k} \leq k_{N C}^{*}$ : in the second case, the conditional level is lower than the level of capital maximising the recipient's utility when BS is granted, $k_{N C}^{*}$. In other words, the conditionality is not binding here and the recipient chooses the capital level $k_{B}^{*}$ that maximizes his utility when both aid modalities are given. This level of capital coincides with the one chosen by the recipient when aid is unconditional:

$k_{B}^{*}=k_{N C}^{*}=\alpha(1-a)(G+B)-(1-\alpha+\alpha a) \gamma A$.

c. when $k_{N C}^{*} \leq \tilde{k} \leq k_{M}$ : the conditional level is greater than the non-conditional level $k_{N C}^{*}$ but still smaller than the $k_{M}$. The conditionality is binding in this case: the recipient chooses the 
capital level $\tilde{k}$. This is so because $V_{C}(k)$ can be shown to be concave. Therefore it is decreasing in $k_{B}^{*}$ and $k_{M}$, as illustrated in figure 1 , and $k \geq k_{M}$ must then bind.

The recipient problem is thus:

$\underset{k_{C}, e, m}{\operatorname{Max}} V\left(m, s\left(k_{C}+\gamma A, e\right)\right)=m^{(1-\alpha)} e^{\alpha a} k_{C}^{\alpha(1-a)}$ s.t. $k_{C}+e+m \leq G+B$ and $k_{N C}^{*} \geq \tilde{k}$

Therefore, we can write the F.O.C. of the maximization problem above as:

$$
\begin{aligned}
& k_{C}^{*}=\left\{\begin{array}{lll}
\operatorname{Max}\left\{\tilde{k}, k_{B}^{*}\right\} & \text { if } & \tilde{k} \leq k_{M} \\
k_{0}^{*} & \text { if } & \tilde{k}>k_{M}
\end{array}\right. \\
& e_{C}^{*}=\frac{\alpha a}{1-\alpha+a \alpha}\left(G+B-k_{C}^{*}\right) \\
& m_{C}^{*}=\frac{1-\alpha}{1-\alpha+a \alpha}\left(G+B-k_{C}^{*}\right) \\
& s_{C}^{*}=\left(\frac{\alpha a}{1-\alpha+\alpha a}\right)^{a}\left(G+B-k_{C}^{*}\right)^{a}\left(k_{C}^{*}+\gamma A\right)^{(1-a)}
\end{aligned}
$$

It is interesting to note at this stage that the individual rationality constraint depends on a level of capital $k_{M}$ that is increasing in the level of the recipient's developmental preferences, $\alpha$, when all the aid is given through budget support:

Lemma 1: $\frac{\partial k_{M}}{\partial \alpha}>0$ when $A=0$ and $B=T$. (Proof in appendix)

The intuition behind this intermediate result is the following: governments whose objectives are far from those of the donor (i.e. governments characterized by a low $\alpha$ ) dislike allocating resources to capital $k$; they prefer $m$, the non-developmental good. Therefore, the IR constraint is harsher for them than for governments whose objectives are closer to the ones of the donors. In other words, governments with a low $\alpha$ satisfy conditionality only if it requires a sufficiently low level of capital. This means in technical terms that they face a lower $k_{M}$.

\section{* The donors' maximisation problem}


We now determine the level of BS, PA and the conditionality threshold maximising the production of the developmental good:

Program 1.

$$
\begin{array}{ll}
\underset{\{A, B, \tilde{k}\}}{\operatorname{Max}} & s_{C}^{*}\left(A, B, k_{C}^{*}\right)=\left(\frac{\alpha a}{1-\alpha+\alpha a}\right)^{a}\left(G+B-k_{C}^{*}\right)^{a}\left(k_{C}^{*}+\gamma A\right)^{1-a} \\
\text { s.t. } & \left\{\begin{array}{l}
A+B=T \\
k_{C}^{*}=\left\{\begin{array}{lll}
\operatorname{Max}\left\{\tilde{k}, k_{B}\right\} & \text { if } & \tilde{k} \leq k_{M} \\
k_{0} & \text { if } & \tilde{k}>k_{M}
\end{array}\right.
\end{array}\right.
\end{array}
$$

We distinguish different candidates for a solution, considering the three cases identified above:

a. If the IR is violated: $k_{C}^{*}=k_{0}=\alpha(1-a) G-(1-\alpha+\alpha a) \gamma A$ when $\tilde{k}>k_{M}$ :

The recipient does not accept the conditional level of capital because it is too high (the IR is violated). Since the conditionality is not respected, no BS will be given: $B=0$. One candidate for a solution in that case is thus:

\section{Result 4:}

$$
\begin{aligned}
& A^{*}=T \text { and } B^{*}=0 ; \\
& k_{0}^{*}=(1-a) \alpha(G+\gamma T)-\gamma T ; \\
& e_{0}^{*}=\alpha a(G+\gamma T) ; \\
& m_{0}^{*}=(1-\alpha)(G+\gamma T) ;
\end{aligned}
$$

The corresponding level of developmental good is therefore

$$
s_{0}^{*}=[\alpha(1-a)(G+\gamma T)-\gamma T]^{1-a} \cdot[\alpha a(G+\gamma T)]^{a} ;
$$

And any $\tilde{k}>(1-a) \alpha(G+\gamma T)-\gamma T$.

b. If the conditional level of capital is smaller than the non-conditional one $\left(\tilde{k}<k_{N C}^{*}\right)$ :

The conditional level of capital is smaller than the one maximising the recipient's utility when no conditionality is imposed. In that case, the conditionality is not a constraint and the donor's 
problem is equivalent to the one solved in Section 4.1. Therefore, all the aid is given through BS and the second candidate for a solution is:

\section{Result 5:}

$$
\begin{aligned}
& A^{*}=0 \text { and } B^{*}=T ; \\
& k_{B}^{*}=\alpha(1-a)(G+T) ; \\
& e_{B}^{*}=\alpha a(G+T) ; \\
& m_{B}^{*}=(1-\alpha)(G+T) ;
\end{aligned}
$$$$
\text { and } s_{B}^{*}=\alpha(1-a)^{1-a} a^{a}(G+T) \text {; }
$$

Any $\tilde{k}<k_{B}^{*}$ is part of the solution.

The level of developmental good $s_{0}^{*}$ is always sub-optimal, because it is always smaller than $s_{B}^{*}$. If the conditionality is too high, that is, for instance, if the donors are too demanding, the introduction of the conditionality yields inefficiency, compared to the case where conditionality is not binding. In fact, if all the aid is given through project aid, the recipient decreases his initial resources allocated to the observable input. This reflects the fungibility problem as explained in the unconditional case. Even if the efficiency loss associated to PA is low, the impact of aid on the development good level will be weaker than with BS (but equivalent if $\gamma=1$ ).

We see that in the two cases above, the introduction of conditionality does not improve the development. In the case where the conditionality is too high, conditionality even harms the development: the level of developmental good $s_{0}^{*}$ is in fact always smaller than $s_{N C}^{*}$ (the level of developmental good when no conditionality is introduced). On the other hand, $s_{B}^{*}$ (the optimal level of developmental good when the conditionality is weak) is equivalent to $s_{N C}^{*}$, since the recipient maximises his utility not regarding the conditionality. Indeed, when the conditional level of capital is smaller than the level maximising the recipient's utility, the introduction of conditionality does not modify the resource allocation, compared to the non-conditional case.

c. If the conditional capital level $\tilde{k}$ is between the non-conditionality level $k_{N C}^{*}$ and the critical level $k_{M}$, the best reply capital level is $\tilde{k}$.

From the F.O.C. of the donors' maximisation problem for this case, we obtain: 


$$
\begin{aligned}
& k_{C}^{*}=\tilde{k}=\operatorname{Min}\left\{(1-a)(T+G), k_{M}\right\} ; \\
& A_{C}^{*}=0 \text { and } B_{C}^{*}=T .
\end{aligned}
$$

The optimal level of conditionality is therefore either the capital level $\tilde{k}=k_{M}$ that binds the IR or $\tilde{k}=(1-a)(G+T)$ that leaves the IR slack and that is equivalent to the level of observable input in the first best allocation.

As can be anticipated from Lemma 1, we prove in Lemma 2 that for high levels of recipient's developmental preferences $\alpha$, the IR is not binding: $(1-a)(T+G)<k_{M}$. The opposite holds for low $\alpha$ : $(1-a)(T+G)>k_{M}$.

Lemma 2: There exists a threshold level $\tilde{\alpha}\left(a, \frac{T}{G}\right)$, decreasing in its arguments, that allows distinguishing the following subcases; relative to the case c above:

(i) For $\alpha>\tilde{\alpha}\left(a, \frac{T}{G}\right)$, a candidate for a solution is $k_{C 1}^{*}=\tilde{k}=(1-a)(T+G) ; A_{C 1}^{*}=0$ and $B_{C l}^{*}=T$. The corresponding level of developmental good is:

$$
s_{C 1}^{*}=(1-a)^{1-a}\left(\frac{\alpha a^{2}}{1-\alpha+\alpha a}\right)^{a}(T+G) .
$$

(ii) For $\alpha<\tilde{\alpha}\left(a, \frac{T}{G}\right)$, a candidate for a solution is $k_{C 2}^{*}=\tilde{k}=k_{M} ; A_{C 2}^{*}=0$ and $B_{C 2}^{*}=T$.

The corresponding level of developmental good is:

$$
s_{C 2}^{*}=\left(\frac{\alpha a}{1-\alpha+\alpha a}\right)^{a}\left(T+G-k_{M}\right)^{a}\left(k_{M}\right)^{1-a} \text {. }
$$

Comparing all the candidates for a solution, we obtain the following result:

\section{Proposition 2:}

The optimal aid modality is always budget support: $B^{*}=T$ and $A^{*}=0$. The optimality of conditionality depends on the developmental preferences of the recipient, the productivity of the 
inputs in the production of the developmental good, and the amount of aid compared to the recipient's government budget:

(i) $\quad$ For $\alpha<\tilde{\alpha}\left(a, \frac{T}{G}\right)$ :

No conditionality needs to be imposed at the optimum (or any level of conditionality that is not binding can be imposed). The resulting allocation of resources is the following:

$$
\begin{aligned}
& k^{*}=\alpha(1-a)(G+T) \quad(\tilde{k}<\alpha(1-a)(G+T)) \\
& e^{*}=\alpha a(G+T) \\
& m^{*}=(1-\alpha)(G+T) \\
& s^{*}=\alpha(1-a)^{1-a} a^{a}(G+T)
\end{aligned}
$$

(ii) For $\alpha>\tilde{\alpha}\left(a, \frac{T}{G}\right)$ :

The optimum requires conditionality: $\tilde{k}=(1-a)(G+T)$. The resulting allocation of resources is the following:

$$
\begin{aligned}
k^{*} & =(1-a)(G+T) \\
e^{*} & =\frac{\alpha a^{2}}{1-\alpha+\alpha a}(G+T) \\
m^{*} & =\frac{a(1-\alpha)}{1-\alpha+\alpha a}(G+T) \\
s^{*} & =(1-a)^{1-a}\left(\frac{\alpha a^{2}}{1-\alpha+\alpha a}\right)^{a}(G+T)
\end{aligned}
$$

The intuition behind proposition 2 is the following: The conditionality should be imposed only on recipients with relatively high development preferences ${ }^{9}$. In fact, when their objectives are relatively close to those of the donors, the introduction of conditionality improves the final production of developmental goods. However, when the preferences are relatively far apart, there is no need to impose conditionality. Indeed, the recipient with low developmental preferences

\footnotetext{
${ }^{9}$ Conditionality is not always optimal, as in Cordella and Dell' Arriccia (2007). If we assume symmetric production function as in their model $(a=1 / 2)$, we have similar results. But it does not hold for other productivity values.
} 
will not respect the resources allocation decided by the donors and consequently they will receive no aid at all. This situation is even worst than a high aid funds eviction to the non-developmental sector. Moreover, even if the conditionality respects the IR, the level of developmental good is lower than when no conditionality is introduced $\left(s_{C 2}^{*}<s_{B}^{*}\right)$. The optimal contract in that case is to give all the aid through unconditional budget support.

It is also interesting to note that in Lemma 2, the threshold level of preferences is decreasing in the aid budget and it is increasing in the recipient's budget. For a recipient with some given developmental preferences, there is a need to condition the allocation of aid as the latter becomes more important relatively to the recipient's own resources. In other words, the need to condition the aid allocation is increasing when the recipient becomes more aid dependent. Moreover, for a given level of preferences, more recipients will be willing to accept the conditionality when the productivity of the observable input $k$ decreases. This reflects the problem of transparency: when the transparency increases, the productivity of the observable input increases. In fact, some unobservable inputs become observable. When the transparency is low (high $a$ ), the level of conditionality imposed by the donors decreases because the observable input $k$ is less productive, but in the same time, more recipients are willing to accept it.

\section{Conclusions}

Foreign aid and its effectiveness in promoting development have been extensively questioned in the literature (see Tarp, 2006 for a survey). In order to understand why aid has not always been effective one must look at the reaction of the recipient government when receiving aid. However, we believe that the modalities chosen to give aid have different impacts on the government incentives to finance the developmental sector. While the existing theoretical literature usually does not consider the existence of different aid disbursement channels, we develop a model considering the two major aid modalities, project aid and budget support. In addition, we allow for the simultaneous utilisation of both modalities, as opposed to Cordella and Dell'Arriccia (2007). In fact, most donors do not have a unified way of giving aid, so that a part of the aid budget is given via BS and another via PA. The interaction of the two modalities also has an impact on the recipient's resources allocation. 
In practice, it is recognised that budget support has a higher impact on development than project aid, because the latter is associated with a loss of efficiency. Indeed, the projects often do not completely fit into the national needs for development (a hospital is built $1 \mathrm{~km}$ away from another one, etc). Moreover, project aid faces the fungiblility problem: the recipient can reallocates some development expenditures to the non-developmental ones having observed the donors' contribution. Budget support has also some limitations: if the corruption is significant, it is clear that the aid is just diverted to non-developmental expenditures and will not foster development.

We have taken these considerations into account to build the analytical framework. First, the model has shown that when no conditionality is enforced, the optimal contract is such that all aid is allocated through budget support and that project aid is not utilised. The fungibility issue is represented by the crowding out of recipient's resources away from the developmental sector. However, when relaxing the hypothesis of loss of efficiency with PA, the donors are indifferent between PA and BS, but the proportion of project aid has to be limited in order to limit the impact of the fungibility. In both cases, since the donors and recipient's preferences are not aligned, there is a distortion in the aid allocation. Some aid funds are indeed diverted to the nondevelopmental sector. The impact of the foreign aid depend thus only on the recipient's preferences. The foreign aid is more efficient when the recipient's preferences are high. For relatively low preferences, the aid has a very weak impact on the production of developmental goods.

We then look at the conditionality as a tool to increase aid effectiveness and derive the optimal contract: the optimal contract depends on the recipient's preferences toward development, the level of aid relative to the recipient's budget and the productivity of the developmental inputs. More specifically, for recipients with high developmental preferences, the optimal aid contract is to give aid only through conditional BS. In that case, the conditionality does improve the level of developmental goods. We have also shown that more recipients accept the conditionality if the transparency decreases and if the aid dependency is relatively high. For relatively low developmental preferences, the optimal contract is such that all the aid is given via unconditional budget support. In fact, the recipients with low development preferences do not accept the conditionality. As seen in the first part, giving the aid through unconditional budget 
support leads to a high distortion in the resources allocation (a relatively large part of the aid is diverted to the non-developmental sector) but it is still better than to give no aid at all.

Consequently, the conditionality does not always improve the aid effectiveness: if the recipient has some low preferences for development, the optimal resources allocation is equivalent to the one of the non-conditional case. It only does for relatively development-oriented recipient: 'More conditionality cannot compensate for weak government commitment or implementation capacity' (World Bank 2005). Our model differs in that sense from the idea that conditionality always improved the development (e.g. Cordella and Dell'Ariccia, 2007). The aid ineffectiveness can thus be reduced only in some situations (high preferences, weak transparency, high aid dependency). This also reflects the idea that weak recipient should not be subject to severer conditions. Even if there is distortion in the aid allocation, it is still better than not helping them at all (François Bourguignon, Le monde, 16.02.2008).

However, the model is based on limited assumptions. A further step will be to compare these two aid disbursement instruments when the recipient's preferences are not observable. Another shortcoming is the implicit assumption of funds predictability. The recipient does not perfectly observe the aid disbursement, especially in the case of project aid. We could introduce this problem of predictability in the model. Our intuition is that the utilisation of PA will increase since the fungibility will reduce.

Last but not least, we would like to comment on the OECD remark about the utility of project aid: 'Within the public sector, projects may be appropriate for mutually agreed activities where a Development Partner is better placed - technically or administratively, to manage the project on behalf of government, for example: technical assistance projects, large scale infrastructure projects, "piloting" projects, where particular service delivery innovations need to be tested before their mainstreaming by government - for example, new approaches to agricultural extension, to road safety or to teaching science.' (OECD, 2007). Our model does not lead to such a conclusion, because we do assume that recipient's and donors' funds are substitutable (if not cost of imperfect fit) and we only allow the possibility that project aid funds are associated with a loss of efficiency. However, we could also consider that in some case (such as the ones mentioned by the OECD), there is a gain in efficiency and not a loss $(\gamma>1)$. Our model also shows that donors could still use project aid to disburse the aid, as soon as no lost of efficiency is associated with the projects. This requires, among others, better coordination with 
the recipient's policies and harmonisation between donors.

\section{References}

Alesina, A. and D. Dollar (2000), "Who gives aid to whom and why?", Journal of Economic Growth 5, 33-63.

Azam, J-P. and J-J. Laffont (2003), "Contracting for Aid" Journal of Development Economics 70(1), 25-58.

Berthélemy, J.C. (2006), “Bilateral donors'interest vs. recipients'development motives in aid allocation: do all donors behave the same?", Review of Development Economics 10, 179194.

Boone, P. (1996), "Politics and the effectiveness of foreign aid", European Economic Review 40(2), 289-329.

Burnside, C. and D. Dollar (2000), "Aid, policies, and growth", American Economic Review 90, 847-68.

Collier, P. and D. Dollar (2002), “Aid allocation and poverty reduction”, European Economic Review 46, 1475-500.

Cordella, T. and G. Dell'Ariccia (2007), "Budget Support Versus Project Aid: a theoretical appraisal", The Economic Journal 117, 1260-1279.

Devarajan S. and V. Swaroop (1998), "The implications of foreign aid fungibility for developmental assistance", Development research group, World Bank, Washington, DC 20433.

Easterly, W. (2003), “Can foreign aid buy growth?”, Journal of Economic Perspectives 17, 23- 
48.

Easterly, W. (2006), “The White Man's Burden: Why the West's Efforts to Aid the Rest Have Done so Much Ill and so Little Good”, Penguin Press, New York.

Hansen, H. and F. Tarp (2001), "aid and growth regressions", Journal of Development Economics 64, 547-70.

Hefeker, C. (2006), "Project Aid or Budget Aid? The interests of Governments and Financial Institutions", Review of Development Economics 10(02), 241-252.

Lavergne, R. (2003), “Les approches-programmes: une nouvelles façon d'opérer”, Direction de la recherche et de l'analyse de l'ACDI.

Mosley P. and M.-J. Eeeckhout (2002), "From project aid to programme assistance", in Foreign aid and Development, Finn Tarp (ed), London, 131-154.

Organisation for Economic Cooperation and Development - Development Assistance Committee (OCDE-DAC) (2003), "Harmonising donor practices for effective aid delivery", DAC reference document, Paris.

Organisation for Economic Cooperation and Development - Development Assistance Committee (OCDE-DAC) (2005), "Paris Declaration on aid effectiveness", available at http://aidharmonisation.org.

Organisation for Economic Cooperation and Development - Development Assistance Committee (OCDE-DAC) (2007), “Joint evaluation of General Budget Support, 1994-2004”, available at http://www.oecd.org/dataoecd.

Rajan R. and A. Subramanian (2005), "What undermines aid's impact on growth?", NBER Working Paper 11657. 
Svensson, J. (2000), "Foreign Aid and Rent-Seeking," Journal of International Economics 51, $437-61$.

Svensson, J. (2003), "Why Conditional Aid Does Not Work and What Can Be Done About It?" Journal of Development Economics 70, 381-402.

Tarp, F. (ed.) (2000), "Foreign Aid and Development: Lessons Learnt and Directions for the Future", Routledge, London and New York.

Tarp, F. (2006),"Aid and Development", Discussion Papers 06-12, University of Copenhagen. Department of Economics .

Torsvik, G. (2005), “Foreign economics aid; should donors cooperated?", Journal of development Economics 77, 503-515.

World Bank, (1998), “Assessing Aid: What Works, What doesn't and why”, Oxford University Press, New York, NY.

World Bank (2005), "Conditionality Revisited", Edited by Stefan Koeberle, Harold Bedoya, Peter Silarsky, and Gero Verheyen. 


\section{Appendix}

\section{Proof of Lemma 1}

The critical level of capital $k_{M}$ is defined such that the level of recipient's utility when budget support is granted and when it is not, are equal : $V_{C}\left(k_{M}\right)=V_{0}\left(k_{0}^{*}\right)$.

We consider the case where $A=O$ and $B=T$.

In that case, $k_{M}$ is such that $f\left(k_{M}, \alpha\right)=0$, where

$f\left(k_{M}, \alpha\right)=\alpha(1-a) \ln \left(k_{M}\right)+(1-\alpha+a \alpha) \ln \left(T+G-k_{M}\right)-\ln (G)-\alpha(1-a) \ln [\alpha(1-a)]-(1-\alpha+a \alpha) \ln (1-\alpha+a \alpha)$.

We determine the sign of the partial derivative of $k_{M}$ with respect to $\alpha$ using the implicit

function theorem: $\frac{\partial k_{M}}{\partial \alpha}=-\frac{\frac{\partial f\left(k_{M}, \alpha\right)}{\partial \alpha}}{\frac{\partial f\left(k_{M}, \alpha\right)}{\partial f\left(k_{M}\right)}}=\frac{(1-a) \ln \left\{1+\frac{k_{M}-k_{B}^{*}}{\alpha(1-a)\left(T+G-k_{M}\right)}\right\}}{\frac{k_{M}-k_{B}^{*}}{k_{M}\left(T+G-k_{M}\right)}}$.

We now prove that this partial derivative is positive.

By construction of $k_{M}$ (see figure 1), we have that $k_{M}>k_{B}^{*}=\alpha(1-a)(T+G)$.

Moreover, the recipient budget constraint $k+e+m \leq G+B$ together with $B=T$ imply $k_{M}<T+G$.

Therefore, at $f\left(k_{M}, \alpha\right)=0$ (which is the equation defining $k_{M}$ ), we have $\frac{\partial k_{M}}{\partial \alpha}>0$.

QED.

\section{Proof of Lemma 2}

We want to determine whether the critical level of capital $k_{M}$ is greater or smaller than the first best level of capital $k_{F B}^{*}=(1-a)(T+G)$. In fact, if $k_{M}>k_{F B}^{*}$, the conditionality is not binding and the optimal level of conditionality is $\tilde{k}=k_{C 1}^{*}=k_{F B}^{*}$. Otherwise, the conditionality is binding and the optimal level of conditionality is to impose the critical level of capital: for $k_{M}<k_{F B}^{*}$, $\tilde{k}=k_{C 2}^{*}=k_{M}$. 
As said in Proof of lemma 1, the critical level of capital $k_{M}$ is such that $f\left(k_{M}, \alpha\right)=0$ and $k_{M}>k_{B}^{*}$. Moreover, $\left.\frac{\partial f(k, \alpha)}{k}\right|_{k=k_{M}}=\frac{k_{B}^{*}-k_{M}}{k_{M}\left(T+G-k_{M}\right)}<0$.

It is thus straightforward that if $f\left(k_{F B}^{*}, \alpha\right)>(<) 0 \Leftrightarrow k_{M}>(<) k_{F B}^{*}$.

We can rewrite $f\left(k_{F B}^{*}, \alpha\right)=\ln \left(1+\frac{T}{G}\right)-g(a, \alpha)$, with

$g(a, \alpha)=-(1-\alpha+\alpha a) \ln \left(\frac{a}{1-\alpha+\alpha a}\right)+\alpha(1-a) \ln (\alpha)$

Moreover, $g(1, \alpha)=g(a, 1)=0$ and $g_{\alpha}^{\prime}(a, \alpha)=(1-a) \ln \left(\frac{\alpha a}{1-\alpha+\alpha a}\right)<0$. The function $g(a, \alpha)$ is also decreasing in the productivity $a$ of the unobservable input: $g_{a}^{\prime}(a, \alpha)=\alpha \ln \left(\frac{1-\alpha+\alpha a}{\alpha a}\right)-\frac{1-\alpha}{a}<0$. Therefore, $g(a, \alpha)>0$.

We can deduce that for low enough values of developmental preferences $\alpha$, $f((1-a)(T+G))=\ln \left(1+\frac{T}{G}\right)-g(a, \alpha)<0$ and then that $k_{M}<(1-a)(T+G)$. The opposite holds for high enough values of $\alpha$. Consequently, there exists a level $\tilde{\alpha}$ such that $f\left(k_{F B}^{*}, \alpha\right)=0$, which allows distinguishing two subcases in Lemma 2. Since $g_{\alpha}^{\prime}<0$ and $g_{a}^{\prime}<0$, the threshold $\tilde{\alpha}$ depends on $\left(\frac{T}{G}, a\right): \tilde{\alpha}\left(\frac{T}{G}, a\right)$, and it is decreasing in its arguments.

QED.

\section{Proof of Proposition 2}

We compare all the candidate solutions. As shown in Lemma 2, we can distinguish 2 subcases.

We begin with the first case: for $\alpha>\tilde{\alpha}\left(a, \frac{T}{G}\right)$. The two possible solutions are either $s_{B}^{*}=\alpha a^{a}(1-a)^{1-a}(T+G) \quad$ with $\quad A_{B}^{*}=0 \quad$ and $B_{B}^{*}=T$ or $s_{C 1}^{*}=(1-a)^{1-a}\left(\frac{\alpha a^{2}}{1-\alpha+\alpha a}\right)(T+G)$ with $A_{C 1}^{*}=0$ and $B_{C 1}^{*}=T$. 
We thus have that $s_{B}^{*}<s_{C 1}^{*} \Leftrightarrow \alpha^{1-a}<\left(\frac{a}{1-\alpha+\alpha a}\right)^{a}$ which is equivalent to the following condition: $\quad h(a, \alpha)=(1-a) \ln (\alpha)-a \ln (a)+a \ln (1-\alpha+\alpha a)<0$. Since $h(1, \alpha)=h(a, 1)=0$ and $h_{\alpha}^{\prime}(\alpha, a)=\frac{(1-a)(1-\alpha)}{\alpha(1-\alpha+\alpha a)}>0$, we have that $h(\alpha, a)<0$ for $\alpha<1$. Consequently, $s_{B}^{*}<s_{C 1}^{*}$. The optimal contract is such that $\left(\tilde{k}^{*}, A^{*}\right)=\left(k_{M}, 0\right)$.

We now turn to the second subcase: for $\alpha<\tilde{\alpha}\left(a, \frac{T}{G}\right)$. There are also two candidates for a solution in this case:

$s_{B}^{*}=\alpha a^{a}(1-a)^{1-a}(T+G)$ with $A_{B}^{*}=0$ and $B_{B}^{*}=T$ or $s_{C 2}^{*}=\left(\frac{\alpha a}{1-\alpha+\alpha a}\right)^{a}\left(T+G-k_{M}\right)^{a}\left(k_{M}\right)^{1-a}$ with $A_{C 2}^{*}=0$ and $B_{C 2}^{*}=T$.

We can now compare the two possible levels of developmental good for this subcase: $s_{C 2}^{*}=\left(\frac{\alpha a}{1-\alpha+\alpha a}\right)^{a}\left(T+G-k_{M}\right)^{a}\left(k_{M}\right)^{1-a}$ and $s_{B}^{*}=\alpha a^{a}(1-a)^{1-a}(T+G)$. From the definition of $k_{M}$, we can write: $\quad\left(s_{C 2}^{*}\right)^{\alpha}=\left(s_{B}^{*}\right)^{\alpha}\left[\frac{(1-\alpha+\alpha a)^{1-\alpha} G}{(T+G)^{\alpha}\left(T+G-k_{M}\right)^{1-\alpha}}\right]$. We can prove that $\frac{(1-\alpha+\alpha a)^{1-\alpha} G}{(T+G)^{\alpha}\left(T+G-k_{M}\right)^{1-\alpha}}<1$. In fact, if $T=0$, then $k=\alpha(1-a) G$ by the definition of $k_{M}$. In that case, $\frac{(1-\alpha+\alpha a)^{1-\alpha} G}{(T+G)^{\alpha}(T+G-k)^{1-\alpha}}=1$ and thus $s_{C 2}^{*}=s_{B}^{*}$. Moreover, $\frac{(1-\alpha+\alpha a)^{1-\alpha} G}{(T+G)^{\alpha}(T+G-k)^{1-\alpha}}$ is decreasing in T. Consequently, we have that $s_{C 2}^{*}<s_{B}^{*}$.

QED. 